\title{
Emodin has a protective effect in cases of severe acute pancreatitis via inhibition of nuclear factor- $k B$ activation resulting in antioxidation
}

\author{
WEI-YAN YAO ${ }^{1}$, YU-FEN ZHOU ${ }^{1}$, AI-HUA QIAN ${ }^{1}$, YONG-PING ZHANG ${ }^{1}$, \\ MIN-MIN QIAO ${ }^{1}$, ZU-KANG ZHAI ${ }^{1}$, YAO-ZONG YUAN ${ }^{1}$ and SONG-LIN YANG ${ }^{2}$
}

\author{
${ }^{1}$ Department of Gastroenterology, Ruijin Hospital, School of Medicine, Shanghai Jiaotong University, Shanghai 200025; \\ ${ }^{2}$ Department of Plastic Surgery, Shanghai Sixth People's Hospital, Shanghai Jiaotong University, Shanghai 200233, P.R. China
}

Received November 15,2013; Accepted June 9, 2014

DOI: $10.3892 / \mathrm{mmr} .2014 .2789$

\begin{abstract}
Severe acute pancreatitis (SAP) accounts for up to $20 \%$ of acute pancreatitis (AP) cases. The absence of effective treatment options has resulted in a high rate of morbidity and mortality. Emodin is a major component of the Chinese herb rhubarb, which has been widely used in the treatment of numerous diseases, including inflammation and cancer. There are a limited number of studies however, that have investigated the effectiveness of emodin in the treatment of SAP. The present study used a rat model of SAP, to investigate the effect and molecular mechanisms of emodin treatment. Administration of emodin was identified to significantly attenuate SAP, as determined by serum amylase analysis and histological assessment of edema, vacuolization, inflammation and necrosis $(\mathrm{P}<0.01)$. Furthermore, treatment with emodin markedly inhibited nuclear factor (NF)- $\kappa$ B DNA-binding activity $(\mathrm{P}<0.01)$ and the serum expression levels of tumor necrosis factor- $\alpha$, interleukin (IL)- 6 and IL-1 $\beta(\mathrm{P}<0.05)$. This attenuation was associated with decreased malondialdehyde and increased superoxide dismutase levels in the pancreatic tissues and serum $(\mathrm{P}<0.05)$. This study indicated that administration of exogenous emodin had therapeutic effects on the severity of SAP. The mechanism may be due to inhibition of $\mathrm{NF}-\kappa \mathrm{B}$ activation resulting in an antioxidation response, which can subsequently suppress the expression of cytokines.
\end{abstract}

Correspondence to: Dr Wei-Yan Yao, Department of Gastroenterology, Ruijin Hospital, School of Medicine, Shanghai Jiaotong University, 197 Ruijin 2nd Road, Shanghai 200025, P.R. China

E-mail: yaowymail@sina.com

Dr Song-Lin Yang, Department of Plastic Surgery, Shanghai Sixth People's Hospital, Shanghai Jiaotong University, 600 Yishan Road, Shanghai 200233, P.R. China

E-mail: alcee@126.com

Key words: severe acute pancreatitis, emodin, antioxidation, nuclear factor- $\kappa \mathrm{B}$

\section{Introduction}

Acute pancreatitis (AP) is one of the most common pancreatic diseases. Although $\sim 80 \%$ of AP can present as a mild self-limiting disorder, severe acute pancreatitis (SAP) accounts for $\sim 20 \%$ of the cases and is associated with a high rate of morbidity and mortality. The mechanisms underlying the disease remain unclear, however, studies have suggested an association with oxidative stress. Upon acinar cell injury, activation of the proinflammatory cascade can lead to local pancreatic necrosis, systemic inflammatory response syndrome as well as distant organ dysfunction in human and experimental animal models (1). Proinflammatory cytokines from activated monocytes and macrophages, such as interleukin (IL)-1 $\beta$, tumor necrosis factor (TNF)- $\alpha$, IL-6 and IL-8, are hypothesized to have an important function in this cascade, and this provides a challenge in the treatment of SAP. The close association between the severity of AP and oxidative stress, suggests that the antioxidant system is a promising therapeutic target for SAP.

Emodin is an anthraquinone compound consisting of three cyclic rings. Emodin is a major component of the widely used Chinese herb, rhubarb, which has various physiological effects, including immunomodulatory (2), antitumor (3), chemopreventive (4) and anti-inflammatory (5) properties. Despite these findings, the potential therapeutic mechanism underlying the effect of emodin in acute pancreatitis has not been addressed.

Nuclear factor (NF)- $\kappa \mathrm{B}$ is a sequence-specific transcription factor known to be involved in the inflammatory and immune response. It functions in numerous physiological and pathologic conditions as an inducible nuclear factor. $\mathrm{NF}-\kappa \mathrm{B}$ mediates various inflammatory mediators involved in acute pancreatitis, including cytokines and adhesion molecules, as well as modulating oxidation. Pyrrolidine dithiocarbamate (PDTC) is a potent inhibitor of $\mathrm{NF}-\kappa \mathrm{B}$, which has been administered to rats to inhibit NF- $\kappa \mathrm{B}$ activation (6). The present study aimed to evaluate the protective roles of emodin and its possible molecular mechanisms of action in SAP. The effects of emodin in a rat model with SAP were investigated, and rats treated with PDTC were used as a control. 


\section{Materials and methods}

Animals and treatment groups. All experiments were conducted with the consent of the University Animal Ethics Committee (Ruijin Hospital, School of Medicine, Shanghai JiaoTong University, Shanghai, China) for the use of Sprague Dawley (SD) rats of the Shanghai Experimental Animals Center of the Chinese Academy of Sciences (Shanghai, China). Healthy male SD rats weighing 220-230 g were housed in standard cages, under climate and temperature $\left(22 \pm 2^{\circ} \mathrm{C}\right)$ control, in a 12-h light/dark cycle. Rats were fed with standard laboratory chow and water ad libitum. The rats were fasted overnight prior to each experiment.

The rats $(n=72)$ were randomly divided into four groups, with each group containing 18 animals: Control group (SO), SAP group, emodin treated group and PDTC treated group. SAP models were established in the SAP, emodin and PDTC treated groups by injection of $200 \mu \mathrm{l}$ of $5 \%$ sterile sodium taurocholate (NaTc; Sigma, St. Louis, MO, USA) into the biliary-pancreatic duct (BPD) for $2 \mathrm{~min}$. The control (SO) group was administered with saline. Pyrrolidine dithiocarbamate (PDTC) was administrated intraperitoneally $1 \mathrm{~h}$ prior to the initiation of pancreatitis at a dose of $100 \mathrm{mg} / \mathrm{kg}$. Emodin was administered once at a dose of $1 \mathrm{mg} / \mathrm{kg}$, following the initiation of pancreatitis. The rats were sacrificed by exsanguination at $1,3,6$, and $12 \mathrm{~h}$, and blood samples and pancreatic tissues were harvested.

The effects of emodin on the survival of SAP-induced rats were assessed $72 \mathrm{~h}$ after the induction of SAP. Healthy rats $(n=30)$ were randomly divided into three groups, with 10 animals per group: SAP group (S), Emodin-treated group and PDTC-treated group. SAP was induced, and PDTC and emodin were administered, as described for the previous groups used in this study.

Serum amylase assay. Serum amylase was determined using a commercially available kit (Randox Laboratories, Crumlin, UK) and by a BeckmanDU 530 Life Science UV/VIS spectrophotometer (Beckman Coulter, Fullerton, CA, USA).

Cytokine assay. Serum TNF- $\alpha$, IL-1 $\beta$ and IL-6 levels were detected by enzyme-linked immunosorbent assays (ELISA) using commercially available kits (Rat Quantikine ELISA kits; R\&D Systems, Minneapolis, MN, USA) according to the manufacturer's instructions. The optical density (OD) was measured using a spectrophotometer at $450 \mathrm{~nm}$ with a correction wavelength set at $570 \mathrm{~nm}$. The sensitivities for TNF- $\alpha$, IL-1 $\beta$ and IL-6 were 15,5 and $5 \mathrm{pg} / \mathrm{ml}$ respectively.

Oxidative stress analyses. Pancreatic tissue was homogenized in ice-cold physiological saline. The superoxide dismutase (SOD) content was measured using a xanthine oxidase technique, based on the spectrophotometric monitoring of SOD-mediated reduction of DTNB at an OD of $550 \mathrm{~nm}$. The concentration of malondialdehyde (MDA) was quantified by a thiobarbituric acid (TBA) reaction, in which MDA or MDA-like substances and TBA react with the production of a pink pigment having an absorption maximum at an OD of $532 \mathrm{~nm}$. SOD and MDA levels were expressed as $\mathrm{nm} / \mathrm{mg}$ protein.
$N F-\kappa B$ DNA-binding assay. DNA-binding affinity was measured using the Trans AM NF- $\kappa$ B Chemi kit (Active Motif, Carlsbad, CA, USA) according to the manufacturer's instructions. Serial dilutions (1:2) were made of the nuclear extracts and $20 \mu \mathrm{l}$ of each dilution and $30 \mu \mathrm{l}$ binding buffer were added to a microtiter plate (Active Motif) coated with an oligonucleotide containing the $\kappa \mathrm{B}$ site of the immunoglobulin (Ig) light chain gene promoter (GGGACTTTCC). The wells were incubated at room temperature for $1 \mathrm{~h}$ and were then washed three times in $200 \mu \mathrm{l}$ washing buffer. Subsequently, the wells were incubated with NF- $\kappa \mathrm{B}$ antibody (1:1,000; Active Motif) for $1 \mathrm{~h}$, and were washed three times in washing buffer. The wells were then incubated for a further $1 \mathrm{~h}$ with horseradish peroxidase-conjugated goat anti-rabbit IgG secondary antibody (1:10,000; Active Motif). The wells were then washed four times, prior to the addition of chemiluminescent substrate (Active Motif). Luminescence was measured using a spectrophotometer set at an OD of $485 \mathrm{~nm}$ with a correction wavelength set at $535 \mathrm{~nm}$.

Western blot analysis. Cells were lysed with radioimmunoprecipitation lysis buffer (Beyotime, Jiangsu, China) supplemented with $1 \mathrm{mM}$ phenylmethanesulfonyl fluoride (Sigma). Anti-NF-кB p65 (phospho S529) antibody was purchased from Abcam (Cambridge, MA, USA). Signals were detected using an Odyssey infrared imaging system (LI-COR, Lincoln, NE, USA) after incubating with IRDye 800 goat anti-rabbit IgG $(\mathrm{H}+\mathrm{L})$ (LI-COR Biosciences, Lincoln, NE, USA) secondary antibodies. Quantification was performed using Image J software (National Institutes of Health, Bethesda, MD, USA).

Histological examination. The tail of the pancreas was fixed in $5 \%$ paraformaldehyde, then dehydrated and embedded in paraffin wax. Sections ( $3-\mu \mathrm{m}$ thick) were cut, dewaxed and stained with hematoxylin and eosin for histological examination. The assessment was performed by an experienced pathologist blind to the experimental design. For the pancreas, a scale of 0 to 4 was used to grade the interstitial edema and hemorrhage, inflammatory cell infiltration, and acinar cell vacuolization and necrosis, consistent with the method described by Schmidt et al (7).

Statistical analysis. The data are presented as the mean \pm standard error of the mean. A one-way analysis of variance was performed by SAS 6.12 (SAS Institute Inc., Cary, NC, USA). Mortality was evaluated using the log rank method. $\mathrm{P}<0.05$ was considered to indicate a statistically significant difference.

\section{Results}

Serum amylase levels. The SAP group showed a marked increase in the level of serum amylase $(\mathrm{P}<0.05)$ after the infusion of $5 \%$ NaTc into the BPD, as compared with the SO group. The application of emodin or PDTC significantly decreased this elevation at each time point (Fig. 1).

Serum cytokine levels. The serum levels of TNF- $\alpha$, IL-1 $\beta$ and IL-6 increased when SAP was induced, whereas the serum levels decreased when emodin or PDTC was administered. In 


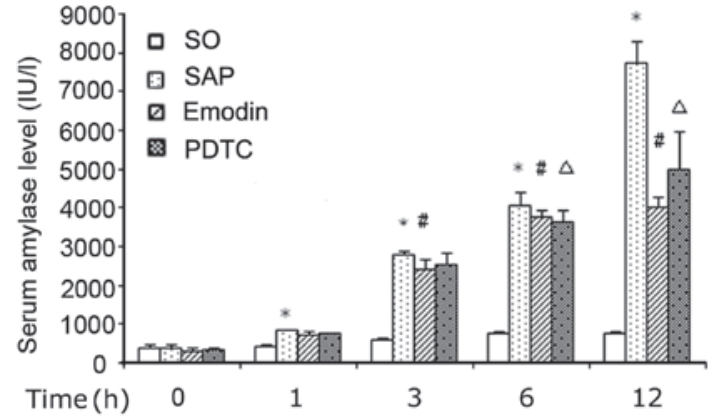

Figure 1. Serum levels of amylase. Serum amylase levels were significantly higher in rats with SAP, as compared with the SO group at 1, 3, 6 and $12 \mathrm{~h}$ $\left({ }^{*} \mathrm{P}<0.05\right)$. The application of emodin attenuated this increase at 3,6 and $12 \mathrm{~h}$ $\left({ }^{\#} \mathrm{P}<0.05\right.$, as compared with SAP group). The application of PDTC attenuated this increase of SAP at 6 and $12 \mathrm{~h}\left({ }^{\triangle} \mathrm{P}<0.05\right.$, as compared with SAP group). SO, control group; SAP, severe acute pancreatitis; PDTC, pyrrolidine dithiocarbamate.

A

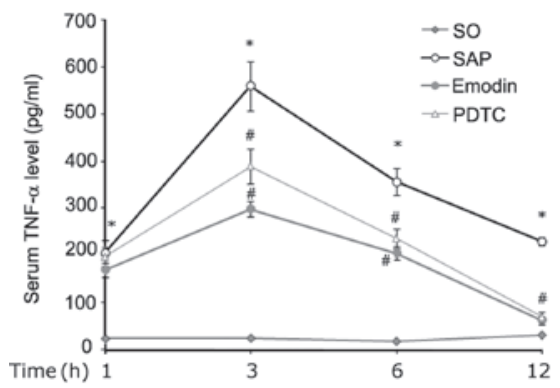

B

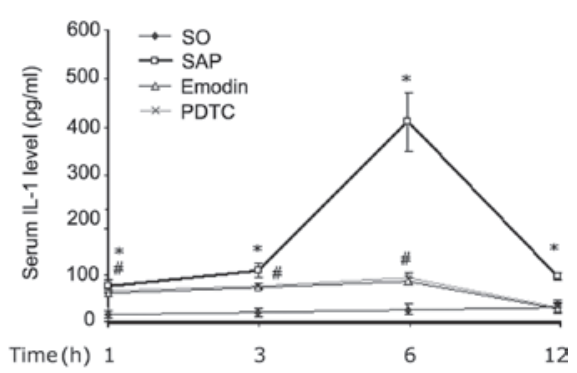

C

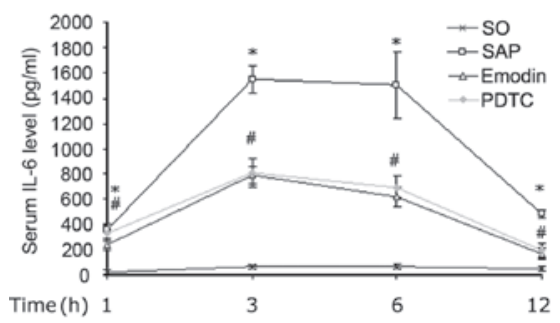

Figure 2. Serum levels of (A) TNF- $\alpha$, (B) IL-1 $\beta$ and (C) IL-6. The induction of SAP increased the serum levels of TNF- $\alpha$, IL- $1 \beta$ and IL- 6 at 1, 3, 6 and $12 \mathrm{~h}\left({ }^{*} \mathrm{P}<0.05\right.$, as compared with the control group). The application of emodin and PDTC markedly decreased TNF- $\alpha$ expression levels at 3,6 and $12 \mathrm{~h}, \mathrm{IL}-1 \beta$ levels at 1,3 and $6 \mathrm{~h}$ and IL- 6 levels at $1,3,6$ and $12 \mathrm{~h}\left({ }^{*} \mathrm{P}<0.05\right.$, as compared with the SAP group). SO, control group; SAP, severe acute pancreatitis; PDTC, pyrrolidine dithiocarbamate; IL, interleukin; TNF- $\alpha$, tumor necrosis factor- $\alpha$.

comparison with the SAP group, the emodin and PDTC treated group had a significantly lower level of TNF- $\alpha$ at 3, 6 and $12 \mathrm{~h}$ $(\mathrm{P}<0.01)$, a lower level of IL-1 $\beta$ at 1,3 and $6 \mathrm{~h}(\mathrm{P}<0.01)$, and $\mathrm{a}$ lower level of IL-6 at 1, 3, 6 and 12 h (Fig. 2).
A

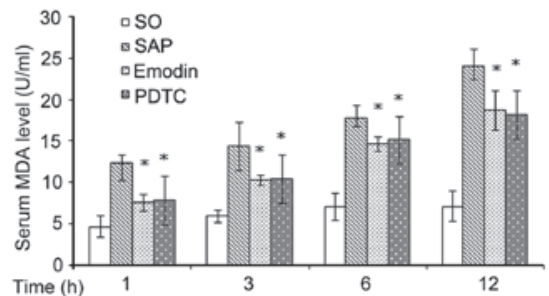

B

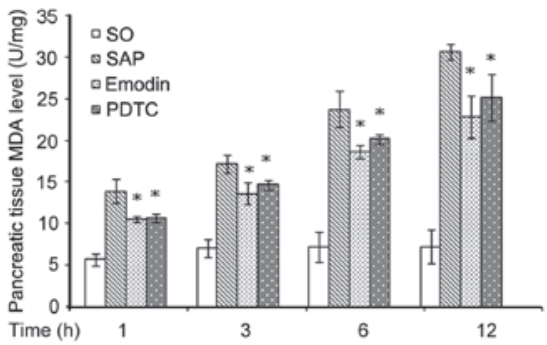

C

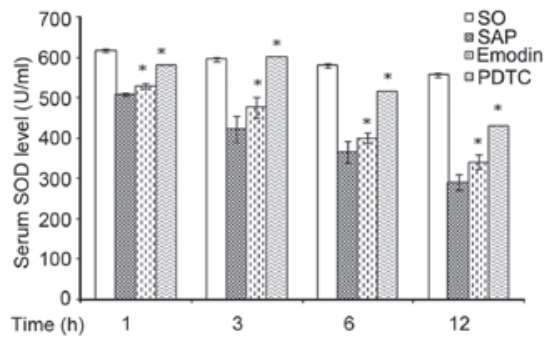

D

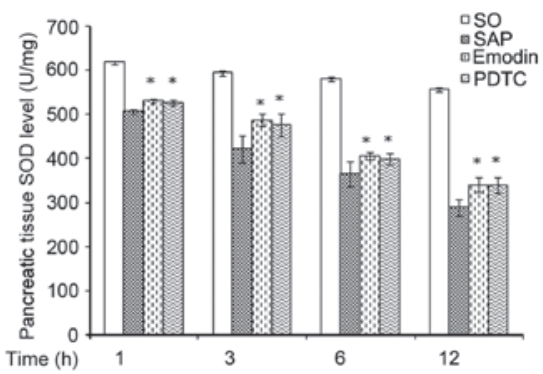

Figure 3. Oxidative stress analyses of (A) MDA levels in the serum and (B) pancreatic tissue, and (C) SOD levels in serum and (D) pancreatic tissue. Induction of SAP markedly increased the activation of NF- $\mathrm{kB}$ in the pancreatic tissue, while application of emodin or PDTC was observed to inhibit this elevation at 3, 6 and $12 \mathrm{~h}$. SO, control group; SAP, severe acute pancreatitis; PDTC, pyrrolidine dithiocarbamate; SOD, superoxide dismutase; MDA, malondialdehyde.

Oxidative stress analyses. There was a significantly higher level of MDA in the pancreatic tissue $(\mathrm{P}<0.05)$, and depleted SOD level $(\mathrm{P}<0.05)$ at each hourly time point investigated in the SAP group. The application of emodin or PDTC markedly decreased the expression levels of MDA and increased the expression level of SOD at 1, 3, 6 and $12 \mathrm{~h}$ (Fig. 3).

$N F-\kappa B$ DNA-binding assay. NF- $\kappa \mathrm{B}$ was shown to be activated in the pancreatic tissue at 1, 3,6 and $12 \mathrm{~h}$ after the induction of SAP. Emodin and PDTC were observed to inhibit this activation (Fig. 4).

$N F-\kappa B$ p 65 western blot assay. NF- $\kappa \mathrm{B}$ p65 protein was detected by western blotting in the pancreatic tissue at 1, 3, 6 and $12 \mathrm{~h}$ after the induction of SAP. Emodin and PDTC were observed to inhibit this activation (Fig. 5). 
Table I. Changes of histological scores.

\begin{tabular}{lcccr}
\hline Group & $1 \mathrm{~h}$ & $3 \mathrm{~h}$ & $6 \mathrm{~h}$ & $1.17 \pm 0.41$ \\
\hline SO & $0.5 \pm 0.55$ & $0.67 \pm 0.52$ & $0.83 \pm 0.41$ & $13.83 \pm 1.17$ \\
SAP & $5.67 \pm 0.52$ & $9.67 \pm 0.52$ & $12.5 \pm 1.05$ & $11.17 \pm 0.98^{\mathrm{a}, \mathrm{b}}$ \\
Emodin & $4.67 \pm 0.52^{\mathrm{a}}$ & $7.67 \pm 0.82^{\mathrm{a}, \mathrm{b}}$ & $10.33 \pm 1.03^{\mathrm{a}, \mathrm{b}}$ & $11.83 \pm 0.75^{\mathrm{a}, \mathrm{b}}$ \\
PDTC & $4.67 \pm 0.52^{\mathrm{a}}$ & $8.67 \pm 0.82^{\mathrm{a}}$ & $10.67 \pm 0.82^{\mathrm{a}, \mathrm{b}}$ &
\end{tabular}

Pancreatic tissue sections were stained with hematoxylin and eosin and examined at $\mathrm{x} 40$ magnification, for damage scoring. The scores were higher in the SAP animals in all categories. The histological score was lower in the rats treated with emodin and PDTC, as compared with the $\mathrm{SAP}$ group. Data are presented as the mean \pm standard deviation for six animals. ${ }^{\mathrm{a}} \mathrm{P}<0.01$ vs. the control group; ${ }^{\mathrm{b}} \mathrm{P}<0.05 \mathrm{vs}$. the $\mathrm{SAP}$ group. SO, control group; SAP, severe acute pancreatitis; PDTC, pyrrolidine dithiocarbamate.

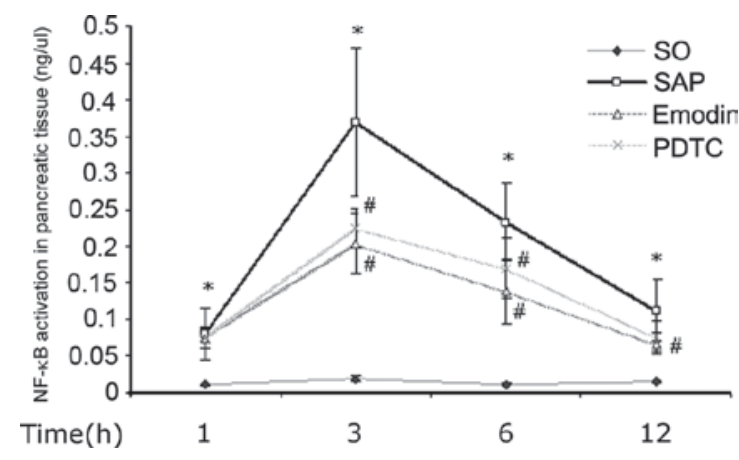

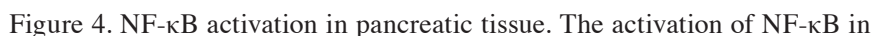
pancreatic tissue was higher after induction of SAP at $1,3,6$ and $12 \mathrm{~h}\left({ }^{*} \mathrm{P}<0.01\right.$, as compared with control group, respectively), while application of emodin or PDTC inhibited this elevation $\left({ }^{*} \mathrm{P}<0.01\right.$, as compared with SAP group, respectively). SO, control group; SAP, severe acute pancreatitis; PDTC, pyrrolidine dithiocarbamate; NF- $\mathrm{\kappa B}$, nuclear factor kappa-light-chain-enhancer of activated B cells.

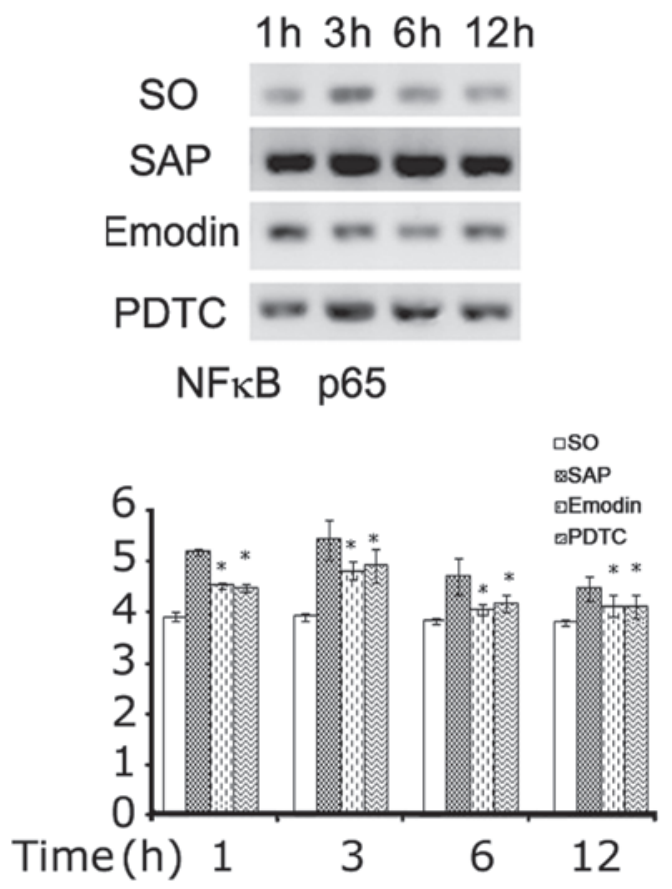

Figure 5. Western blot analysis of NF-кB p65 was detected in the pancreatic tissue at 1,3, 6 and $12 \mathrm{~h}$ after the induction of SAP, while application of emodin or PDTC inhibited NF- $\kappa \mathrm{B}$ p65 elevation. ${ }^{*} \mathrm{P}<0.01$, as compared with the control group. Data are presented as the mean \pm standard deviation. $\mathrm{SO}$, control group; SAP, severe acute pancreatitis; PDTC, pyrrolidine dithiocarbamate.

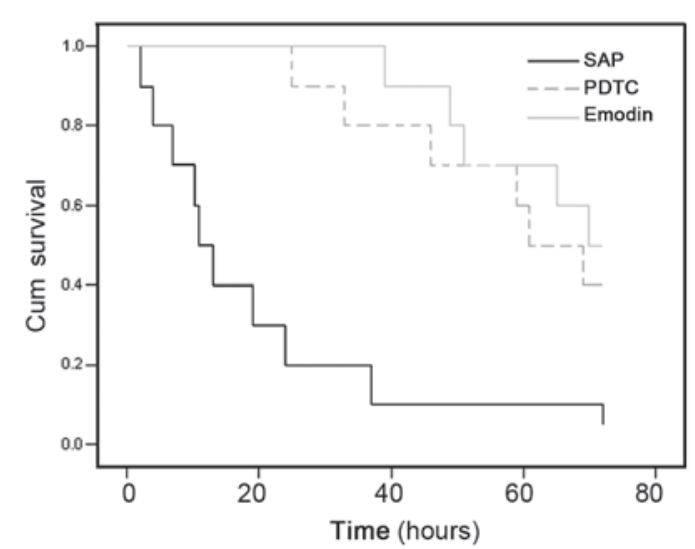

Figure 6. Kaplan-Meier survival curves. The survival rate of rats with severe acute pancreatitis was significantly improved, upon treatment with emodin or PDTC. The most marked effect was observed in animals treated with emodin. Only one animal survived to the end of the experiment $(72 \mathrm{~h}$ ) in the SAP group.

Histological findings. The grade of pancreatitis was evaluated based on the Schmidt histological scoring system (5). Large areas of necrosis, together with infiltration of inflammatory cells and hemorrhage, were identified in the SAP group, which had a higher histological score as compared with the control group (Table I). Following emodin and PDTC treatment, less inflammation and hemorrhage was observed, and necrosis was detected in $<30 \%$ of the total areas.

Survival. The majority of the SAP rats died within two days when no treatment was applied. By contrast, emodin or PDTC could significantly improve the survival rate, with $40 \%$ of the rats still alive at the end of the study (three days) (Fig. 6).

\section{Discussion}

The present study used an experimental SAP rat model to determine whether emodin could significantly improve the oxidative stress and morphological injury in pancreatic tissues. Previous studies have indicated that emodin may influence antioxidant function, cytokine production and TGF signaling, which has been demonstrated in an ischemia injury model (8). Furthermore, it has been proposed that emodin could enhance tissue regeneration through the regulation of the Smad-TGF- $\beta 1$ pathway (9). Few studies have focused on the effects of emodin on SAP. A reliably induced model with severe, acute onset 
of hemorrhagic and necrotic pancreatitis, was established in rats by retrograde infusion of NaTc in the BPD. It was subsequently shown that emodin could decrease the serum levels of TNF- $\alpha$ and IL-1 $\beta$, thus improving the morphological damage and survival rate. Similar results have been reported demonstrating the effective treatment of clinical and experimental acute pancreatitis by emodin (10-12).

It has been shown that significant lipid peroxidation occurs in AP $(13,14)$. Reactive oxygen species can activate $\mathrm{NF}-\kappa \mathrm{B}$, which can in turn regulate the expression of inflammatory cytokines, such as TNF- $\alpha$ and ILs in acinar cells, and lead to the activation of a cascade of inflammatory cytokines (15). This is hypothesized to function in the pathogenesis and the progression of acute pancreatitis and the systemic complications. PDTC is commonly used as an antioxidant, which can regulate gene expression by modulating the activity of transcription factors (16). PDTC can block NF- $\kappa \mathrm{B}$ from binding to its target DNA by preventing the degradation of I $\kappa$ B- $\alpha$ from the ubiquitylation-proteasome proteolytic pathway. $\mathrm{NF}-\kappa \mathrm{B}$ is known to regulate numerous proinflammatory molecules, including IL-6, IL-1, TNF- $\alpha$, and inducible nitric oxide synthase, cell surface adhesion molecules (such as E-selectin, intercellular adhesion molecule-1 and vascular cell adhesion molecule-1) and cell surface mediators (such as major histocompatibility complexes class I and II) through $\mathrm{NF}-\kappa \mathrm{B}$ binding sites in their promoter regions. It has been shown that antioxidant treatment could inactivate $\mathrm{NF}-\kappa \mathrm{B}$ and inhibit the activation of neutrophils in acute pancreatitis (17). In the present study, treatment with PDTC significantly attenuated SAP, as determined by serum amylase and histological assessment of edema, vacuolization, inflammation and necrosis $(\mathrm{P}<0.01)$. Furthermore, treatment with PDTC markedly inhibited NF- $\kappa$ B DNA-binding activity $(\mathrm{P}<0.01$, as compared with the SAP group) and the expression of TNF- $\alpha$, IL- 6 and IL-1 in the localized tissues and the serum $(\mathrm{P}<0.05)$. The observed attenuation was associated with decreased MDA and increased SOD levels, in the pancreatic tissues and serum $(\mathrm{P}<0.05)$. This is consistent with previous studies investigating other inflammatory diseases (18-20). In the present study, treatment with emodin had a similar effect to treatment with PDTC. Emodin could inhibit $\mathrm{NF}-\kappa \mathrm{B}$ activation and regulate cytokine production, thereby regulating the oxidative stress response and improving the histological changes and survival rate. In conclusion, this study has shown that in an experimental rat model of SAP, emodin treatment significantly improved the oxidative stress and morphological injury in the pancreatic tissue, suggesting antioxidant treatment strategies may have clinical benefits. Understanding the therapeutic effects of emodin in AP and SAP, and its mechanisms of action is likely to guide future studies in this field.

\section{Acknowledgements}

This study was supported by the National Scientific Fund (grant no. 81101849).

\section{References}

1. Makhija R and Kingsnorth AN: Cytokine storm in acute pancreatitis. J Hepatobiliary Pancreat Surg 9: 401-410, 2002.

2. Fujimoto H, Nakamura E, Okuyama E and Ishibashi M: Six immunosuppressive features from an ascomycete, Zopfiella longicaudata, found in a screening study monitored by immunomodulatory activity. Chem Pharm Bull (Tokyo) 52: 1005-1008, 2004.

3. Srinivas G, Babykutty S, Sathiadevan PP and Srinivas P: Molecular mechanism of emodin action: transition from laxative ingredient to an antitumor agent. Med Res Rev 27: 591-608, 2007.

4. Braumann C, Tangermann J, Jacobi CA, Muller JM and Dubiel W: Novel anti-angiogenic compounds for application in tumor therapy - COP9 signalosome-associated kinases as possible targets. Mini Rev Med Chem 8: 421-428, 2008.

5. Wu Y, Tu X, Lin G, et al: Emodin-mediated protection from acute myocardial infarction via inhibition of inflammation and apoptosis in local ischemic myocardium. Life Sc 81: 1332-1338, 2007.

6. Yucel M, Kucuk A, Bayraktar AC, Tosun M, Yalcinkaya S, Hatipoglu NK, Erkasap N and Kavutcu M: Protective effects of the nuclear factor kappa B inhibitor pyrrolidine dithiocarbamate in bladder ischemia-reperfusion injury in rats. Mol Biol Rep 40: 5733-5740, 2013.

7. Schmidt J, Lewandrowsi K, Warshaw AL, Compton CC and Rattner DW: Morphometric characteristics and homogeneity of a new model of acute pancreatitis in the rat. Int J Pancreatol 12: 41-51, 1992

8. Lu JS, Liu JX, Zhang WY, et al: Preventive effects of emodin on cerebral ischemia injury and expression of the inflammatory factors in rats with cerebral ischemia. Zhongguo Zhong Yao Za Zhi 30: 1939-1943, 2005 (In Chinese)

9. Tang T, Yin L, Yang J and Shan G: Emodin, an anthraquinone derivative from Rheum officinale Baill, enhances cutaneous wound healing in rats. Eur J Pharmacol 567: 177-185, 2007.

10. Wang G, Sun B, Gao Y, Meng QH and Jiang HC: An experimental study of emodin assisted early enteral nutrition for the treatment of severe acute pancreatitis. Hepatogastroenterology 55: 33-40, 2008.

11. Gong Z, Yuan Y, Lou K, et al: Mechanisms of Chinese herb emodin and somatostatin analogs on pancreatic regeneration in acute pancreatitis in rats. Pancreas 25: 154-160, 2002.

12. Wu JX, Xu JY and Yuan YZ: Effect of emodin and sandostatin on metabolism of eicosanoids in acute necrotizing pancreatitis. World J Gastroenterol 6: 293-294, 2000.

13. Gutierrez PT, Folch-Puy E, Bulbena O and Closa D: Oxidised lipids present in ascitic fluid interfere with the regulation of the macrophages during acute pancreatitis, promoting an exacerbation of the inflammatory response. Gut 57: 642-648, 2008.

14. Abu-Hilal M, McPhail MJ, Marchand L and Johnson CD: Malondialdehyde and superoxide dismutase as potential markers of severity in acute pancreatitis. JOP 7: 185-192, 2006.

15. Ji LL, Gomez-Cabrera MC and Vina J: Role of nuclear factor kappaB and mitogen-activated protein kinase signaling in exercise-induced antioxidant enzyme adaptation. Appl Physiol Nutr Metab 32: 930-935, 2007.

16. Mingyan E, Hongli L, Shufeng L and Bo Y: Effects of pyrrolidine dithiocarbamate on antioxidant enzymes in cardiomyopathy induced by adriamycin in rats. Cardiology 111: 119-125, 2008.

17. Surh YJ: NF-kappa B and Nrf2 as potential chemopreventive targets of some anti-inflammatory and antioxidative phytonutrients with anti-inflammatory and antioxidative activities. Asia Pac J Clin Nutr 17: 269-272, 2008.

18. Jaworek J, Nawrot-Porabka K, Leja-Szpak A, et al: Melatonin as modulator of pancreatic enzyme secretion and pancreatoprotector. J Physiol Pharmacol 58: 65-80, 2007.

19. Lawinski M, Sledzinski Z, Kubasik-Juraniec J, et al: Does resveratrol prevent free radical-induced acute pancreatitis? Pancreas 31: 43-47, 2005.

20. Wereszczynska-Siemiatkowska U, Mroczko B, Siemiatkowski A, et al: The importance of interleukin 18, glutathione peroxidase, and selenium concentration changes in acute pancreatitis. Dig Dis Sci 49: 642-650, 2004. 